\title{
Impacto da estrutura de detenção na ocorrência de infecção por SARS-CoV-2 em jovens detentos
}

\author{
Impact of detention structure on the occurrence of SARS-CoV-2 infection in young inmates
}

Impacto de la estructura de detención en la incidencia de la infección por SARS-CoV-2 en reclusos

jóvenes

Recebido: 13/12/2021 | Revisado: 18/12/2021 | Aceito: 20/12/2021 | Publicado: 03/01/2022

Lysandro Pinto Borges

ORCID: https://orcid.org/0000-0002-1721-1547 Universidade Federal de Sergipe, Brasil E-mail: lysandro.borges@gmail.com Adriana Gibara Guimarães ORCID: https://orcid.org/0000-0003-1643-5642 Universidade Federal de Sergipe, Brasil E-mail: adrianagibara@hotmail.com

Daniela Raguer Valadão de Souza ORCID: https://orcid.org/0000-0001-9897-9701 Universidade Federal de Sergipe, Brasil

E-mail: daniraguer@gmail.com

Aline Fagundes Martins

ORCID: https://orcid.org/0000-0001-9255-6716

Universidade Federal de Sergipe, Brasil

E-mail: draalinemartins.orto@ gmail.com

José Melquiades de Rezende Neto ORCID: https://orcid.org/0000-0002-4955-8529

Universidade Federal de Sergipe, Brasil E-mail: jmkide@gmail.com

Aryanne Araujo Santos

ORCID: https://orcid.org/0000-0002-7342-8927 Universidade Federal de Sergipe, Brasil

E-mail: ary_anne10@hotmail.com

Grazielly Bispo da Invenção

ORCID: https://orcid.org/0000-0003-4137-8010 Universidade Federal de Sergipe, Brasil

E-mail: graziellyinvencao@hotmail.com

Igor Leonardo Santos Matos

ORCID: https://orcid.org/0000-0001-9165-383X Universidade Federal de Sergipe, Brasil E-mail: igor_leonardo100@hotmail.com

Kezia Alves dos Santos

ORCID: https://orcid.org/0000-0003-4669-4792 Universidade Federal de Sergipe, Brasil

E-mail: keeziaalves@gmail.com

Nicolas Alessandro Alves Souza

ORCID: https://orcid.org/0000-0003-4328-8369 Universidade Federal de Sergipe, Brasil E-mail: nicolasalessandro@gmail.com

Cristiani Isabel Banderó Walker

ORCID: https://orcid.org/0000-0001-6219-2325 Universidade Federal de Sergipe, Brasil

E-mail: bandewalk@hotmail.com

Pamela Chaves de Jesus

ORCID: https://orcid.org/0000-0003-3282-7056 Universidade Federal de Sergipe, Brasil

E-mail: pamcjesus@outlook.com

Lylyan Gleyze Brito de Oliveira

ORCID: https://orcid.org/0000-0002-1834-988X

Fundação Renascer do Estado de Sergipe E-mail: lylyangleyze@yahoo.com.br

Makson Gleydson Brito de Oliveira

ORCID: https://orcid.org/0000-0002-3668-012X Universidade Federal de Sergipe, Brasil E-mail: makson_gbo@hotmail.com 


\begin{abstract}
Resumo
Considerando que a COVID-19 é uma doença respiratória transmitida por contato e os locais de encarceramento são um ambiente que impõe riscos adicionais para a disseminação viral, este estudo buscou identificar os casos de infecção por SARS-CoV-2 em unidades socioeducativas. Foi avaliada a soroprevalência de $\operatorname{IgM}$ e $\operatorname{IgG}$ contra o SARS-CoV-2 em trabalhadores e adolescentes confinados em cinco unidades socioeducativas. Adolescentes e trabalhadores testaram positivo para $\operatorname{IgM}(n=8$, cada), $\operatorname{IgG}(n=26 ; n=6)$ e ambos $(n=13 ; n=6)$, com maior chance de detectar IgG (OR: 2,16, p = 0,03) e ambos (OR: 68,59, p <0,0001) em adolescentes que viviam em acomodação compartilhada (unidade 5) do que aqueles que viviam em quartos separados (unidades 1-4). O estudo evidenciou a prevalência de COVID-19 em uma comunidade socioeducativa, onde o tipo de moradia ocupada pelos indivíduos (cômodo simples x dormitório) foi o principal fator determinante da disseminação da doença. Mesmo adolescentes em confinamento apresentaram anticorpos para SARS-CoV-2.
\end{abstract}

Palavras-chave: SARS-CoV-2; COVID-19; Unidades socioeducativas; Ensaios de imunofluorescência.

\begin{abstract}
Considering that COVID-19 is a respiratory disease transmitted by contact and places of incarceration are an environment that imposes additional risks for viral spread, this study sought to identify cases of SARS-CoV-2 infection in socio-educational units. We evaluated the seroprevalence of IgM and IgG for SARS-CoV-2 in workers and adolescents confined in five socio-educational units. Adolescents and workers tested positive for $\operatorname{IgM}(\mathrm{n}=8$, each), $\operatorname{IgG}(n=26 ; n=6)$ and both $(n=13 ; n=6)$, with a greater chance of detecting $\operatorname{IgG}(\mathrm{OR}: 2.16, \mathrm{p}=0.03)$ and both (OR: $68.59, \mathrm{p}<0.0001$ ) in adolescents who lived in shared accommodation accommodation (unit 5) than those who lived in separate rooms (units 1-4). The study showed the prevalence of COVID-19 in a socio-educational community, where the type of housing occupied by the individuals (single rooms vs a dormitory) was the main determining factor or the spread of the disease. Even adolescents in confinement presented antibodies to SARS-CoV-2.
\end{abstract}

Keywords: SARS-CoV-2; COVID-19; Socio-educational units; Immunofluorescence assays.

\title{
Resumen
}

Considerando que COVID-19 es una enfermedad respiratoria transmitida por contacto y los lugares de encarcelamiento son un ambiente que impone riesgos adicionales de propagación viral, este estudio buscó identificar casos de infección por SARS-CoV-2 en unidades socioeducativas. Evaluamos la seroprevalencia de IgM e IgG para SARS-CoV-2 en trabajadores y adolescentes confinados en cinco unidades socioeducativas. Los adolescentes y trabajadores dieron positivo para $\operatorname{IgM}(n=8$, cada $u n o), \operatorname{IgG}(n=26 ; n=6)$ y ambos $(n=13 ; n=6)$, con una mayor probabilidad de detectar IgG (OR: 2,16, p = 0,03) y ambos (OR: 68,59, p <0,0001) en adolescentes que vivían en alojamiento compartido (unidad 5) que en los que vivían en habitaciones separadas (unidades 1-4). El estudio mostró la prevalencia de COVID-19 en una comunidad socioeducativa, donde el tipo de vivienda ocupada por los individuos (habitaciones individuales vs dormitorio) fue el principal factor determinante de la propagación de la enfermedad. Incluso los adolescentes confinados presentaron anticuerpos contra el SARS-CoV-2.

Palabras clave: SARS-CoV-2; COVID-19; Unidades socioeducativas; Ensayos de inmunofluorescencia.

\section{Introdução}

Ao longo dos anos de 2020 e 2021, a pandemia do coronavírus 2019 (COVID-19) expandiu-se rapidamente, afetando todos os setores da sociedade (Borges et al., 2021; CDC, 2020; Diamanti \& Nikolaou, 2021). O surto do SARS-CoV-2, também chamado novo coronavírus 2, iniciou em dezembro de 2019 em Wuhan na China e em poucos meses disseminou-se ao redor do mundo, sendo reconhecida como pandemia pela Organização Mundial da Saúde (OMS), apresentando elevadas taxas de infecção e mortes (Huang et al., 2020; WHO, 2020; Zhu et al., 2020).

A COVID-19 é uma doença de origem respiratória e sua transmissão depende principalmente do contato com secreções respiratórias entre os indivíduos. Deste modo, locais de encarceramento de indivíduos em conflito com a lei são extremamente suscetíveis à disseminação rápida e descontrolada de doenças infecciosas, conforme relatado na literatura a respeito da disseminação histórica de influenza, tuberculose e outros patógenos respiratórios (CDC, 2020; Maruschak et al., 2009).

Estes tipos de ambientes impõem riscos adicionais para a disseminação viral, devido à superlotação, má ventilação e por se tratarem de ambientes fechados (CDC, 2020; Guo et al., 2019). Além disso, o baixo nível socioeconômico, baixo nível de educação e sexo masculino foram caracterizados como fatores de risco para transmissão da SARS-CoV-2, características que se refletem entre os encarcerados (Madahar et al., 2021). Assim, é urgente monitorar a prevalência de COVID-19 nesse 
tipo de população e adotar medidas preventivas rigorosas para reduzir a chance da doença se espalhar rapidamente em ambiente confinado (Borges et al., 2021; Carvalho et al., 2020).

No Brasil, adolescentes condenados por crime (ou que aguardam julgamento) são colocados sob "medidas socioeducativas" e confinados em centros de detenção juvenil, sendo privados de viver em sociedade. A legislação que regulamenta os direitos da criança e do adolescente (Estatuto da Criança e do Adolescente), bem como a política de implementação de medidas socioeducativas, preveem a atenção integral à saúde do adolescente, conforme art. 60 da Lei 12.594, de 18 de janeiro de 2012 (Sistema Nacional de Atendimento Social e Educacional) (Brasil, 2021). No estado de Sergipe, a Fundação Renascer, por meio de seus centros de internação juvenil, é responsável pelo atendimento a essas questões.

Por estas razões, o objetivo deste estudo foi avaliar a soroprevalência de IgM e IgG contra o SARS-CoV-2 em funcionários e adolescentes internados em cinco unidades socioeducativas do estado de Sergipe, Brasil.

\section{Metodologia}

Foi realizado um estudo observacional de natureza epidemiológica com corte transversal, conforme a metodologia STROBE (Strengthening the Reporting of Observational Studies in Epidemiology) (von Elm et al., 2008) em cinco unidades socioeducativas, nos municípios de Aracaju (Unidades 1, 2, 3 e 4) e Nossa Senhora do Socorro (Unidade 5), as quais fazem parte da Fundação Renascer do Estado de Sergipe. Em agosto de 2020, os jovens detentos e funcionários do corpo administrativo de cada unidade foram inicialmente apresentados ao projeto e, após aceitarem participar do estudo, assinaram o termo de consentimento livre e esclarecido, em conformidade com as normas éticas em pesquisa com seres humanos desenvolvidos no Brasil (Brasil, 2012) e aprovação pelo CEP/UFS (Parecer: 4296250). Os dados pessoais, incluindo idade, sexo, endereço, presença de quaisquer comorbidades e sintomas compatíveis com COVID-19, foram coletados por meio de um questionário online conduzido por um analista clínico farmacêutico antes da coleta de sangue.

Aproximadamente $3 \mathrm{~mL}$ de sangue foram coletados de cada voluntário. A punção venosa foi a técnica escolhida, com dois possíveis locais de coleta: a fossa antecubital do braço ou o dorso da mão. As amostras foram armazenadas em um ambiente refrigerado para manter propriedades biológicas viáveis para o teste de anticorpos IgG e IgM para SARS-CoV-2. No laboratório, a amostra de sangue total foi centrifugada a $4000 \mathrm{rpm}$ por 15 minutos. O soro foi armazenado em tubos Eppendorf ${ }^{\circledR}$ e mantido refrigerado a $-20^{\circ} \mathrm{C}$ até o momento do teste. Este material biológico foi analisado por meio do teste Ichroma ${ }^{\circledR}$ COVID-19 Ab (Boditech Med Inc., Chuncheon, Coréia), seguindo as instruções do fabricante para a detecção de anticorpos IgG e IgM para SARS-CoV-2 pelo método de fluorescência.

Os ensaios de imunofluorescência foram realizados no Laboratório do Departamento de Farmácia (Laboratório de Bioquímica e Imunologia Clínica, LaBiC-Imm) da Universidade Federal de Sergipe (UFS). Os anticorpos anti-SARS-CoV-2 IgM e IgG foram detectados no soro usando um sistema de teste de diagnóstico in vitro baseado na tecnologia de imunofluorescência de detecção de sanduíche de fluxo lateral (Ichroma ${ }^{\mathrm{TM}}$ II COVID-19 Ab, em conjunto com um Ichroma ${ }^{\mathrm{TM}}$ II Reader, Boditech Med Inc., Coreia do Sul) de acordo com as instruções do fabricante. O método de imunofluorescência aplicado apresentou sensibilidade de $95,8 \%$ e especificidade de $97 \%$, conforme estudos de validação prévios realizados pelo nosso grupo de pesquisa (Borges et al., 2020; Melo et al., 2020).

Os dados obtidos foram analisados por meio de estatística descritiva e expressos em dados brutos e percentuais. Usando o Microsoft Excel (2013), estimamos a linha de tendência a partir de uma tendência linear simples, usando análise de regressão. As associações entre soropositividade e localização das unidades foram avaliadas por meio do teste exato de Fisher

e GraphPad Prism ${ }^{\circledR} 6.0$ (GraphPad Software, Inc., CA. USA), por meio da estimativa do Odds ratio (OR) com intervalo de confiança de 95\% (IC 95\%). O nível de significância estatística utilizado foi p $<0,05$. 


\section{Resultados e Discussão}

A Fundação Renascer do Estado de Sergipe, instituição pública vinculada à Secretaria de Inclusão e Assistência Social do Estado de Sergipe, é responsável pela implementação da Política Estadual de atendimento e proteção à criança e adolescente em situação de risco pessoal e social. A fundação é responsável pelos Centros de Internação Juvenil do Estado, que atendem adolescentes de 12 a 21 anos. Trabalha para o retorno dos adolescentes ao convívio social e familiar, além de garantir seus direitos previstos em lei, entre sendo eles a garantia do direito à saúde. Assim, este estudo utilizou testes diagnósticos COVID-19 rápidos e precisos para avaliar os níveis de infecção da doença.

Foram testados 301 participantes da comunidade socioeducativa da Fundação Renascer do Estado de Sergipe. Destes, 200 eram adolescentes em confinamento e 101 eram trabalhadores da Fundação Renascer de diferentes setores administrativos (Tabela 1). A amostra de adolescentes foi composta predominantemente por jovens do sexo masculino $(\mathrm{n}=191 ; 95,5 \%)$, com idade entre 15 e 18 anos $(n=133 ; 66,5 \%)$. Alguns adolescentes relataram a presença de comorbidades como: asma, alergias, dislipidemia e HIV. A presença de sintomas gripais também foi relatada por aproximadamente 30 jovens (dados não mostrados). Entre os trabalhadores das unidades, também houve a predominância do sexo masculino ( $\mathrm{n}=70 ; 69,3 \%)$, sendo a maioria com idade superior a 40 anos $(n=54,53,5 \%)$. Este grupo também relatou a presença de comorbidades, com destaque para diabetes e hipertensão arterial sistêmica. Cerca de 10 trabalhadores referiram a presença de sintomas semelhantes aos da gripe (dados não mostrados).

Tabela 1: Perfil demográfico e soroprevalência de IgM e IgG contra SARS-CoV-2 em cinco centros de internação juvenil (15) no estado de Sergipe, Brasil.

\begin{tabular}{|c|c|c|c|c|c|c|c|}
\hline \multirow{2}{*}{ Adolescentes } & \multicolumn{5}{|c|}{ Unidades } & \multirow{2}{*}{ Total } & \multirow{2}{*}{$\%$} \\
\hline & 1 & 2 & 3 & 4 & 5 & & \\
\hline $\mathrm{n}$ & 9 & 10 & 9 & 92 & 80 & 200 & 100 \\
\hline Masculino & 0 & 10 & 9 & 92 & 80 & 191 & 95,5 \\
\hline Feminino & 9 & 0 & 0 & 0 & 0 & 9 & 4,5 \\
\hline \multicolumn{8}{|l|}{ Faixa etária } \\
\hline$<15$ & 0 & 0 & 0 & 5 & 4 & 9 & 4,5 \\
\hline $15-18$ & 7 & 7 & 3 & 67 & 49 & 133 & 66,5 \\
\hline $18>$ & 2 & 3 & 6 & 19 & 27 & 57 & 28,5 \\
\hline Não informado & 0 & 0 & 0 & 1 & 0 & 1 & 0,5 \\
\hline IgM reagente & 1 & 1 & 1 & 3 & 2 & 8 & 4,0 \\
\hline IgG reagente & 0 & 0 & 0 & 4 & 22 & 26 & 13,0 \\
\hline IgM e IgG reagente & 0 & 0 & 0 & 0 & 13 & 13 & 6,5 \\
\hline Não reagente & 8 & 9 & 8 & 85 & 43 & 153 & 76,5 \\
\hline \multirow{2}{*}{ Trabalhadores } & \multicolumn{5}{|c|}{ Unidades } & \multirow{2}{*}{ Total } & \multirow{2}{*}{$\%$} \\
\hline & 1 & 2 & 3 & 4 & 5 & & \\
\hline $\mathrm{n}$ & 6 & 8 & 6 & 51 & 30 & 101 & 100 \\
\hline Masculino & 1 & 6 & 3 & 39 & 21 & 70 & 69,3 \\
\hline Feminino & 5 & 2 & 3 & 12 & 9 & 31 & 30,7 \\
\hline \multicolumn{8}{|l|}{ Faixa etária } \\
\hline$<30$ & 0 & 0 & 0 & 1 & 2 & 3 & 3,0 \\
\hline $30-40$ & 6 & 4 & 2 & 20 & 12 & 44 & 43,6 \\
\hline$>40$ & 0 & 4 & 4 & 30 & 16 & 54 & 53,5 \\
\hline IgM reagente & 1 & 0 & 0 & 4 & 3 & 8 & 7,9 \\
\hline IgG reagente & 0 & 0 & 1 & 2 & 3 & 6 & 5,9 \\
\hline IgM e IgG reagente & 0 & 0 & 0 & 3 & 3 & 6 & 5,9 \\
\hline Não reagente & 5 & 8 & 5 & 42 & 21 & 81 & 80,2 \\
\hline
\end{tabular}


Dos 200 adolescentes, $8(4,0 \%)$ apresentaram IgM positivo, $26(13,0 \%) \operatorname{IgG}$ positivo, $13(6,5 \%)$ apresentaram soro reagente para IgM e IgG e 153 jovens (76,5\%) não apresentaram nenhum tipo de anticorpo estudado contra o coronavírus. A unidade 5 se destacou pela proporção de adolescentes em que foram detectados IgG ou ambos os anticorpos, apresentando razão de chance 2,16 ( $\mathrm{p}=0,03)$ e 68,59 ( $\mathrm{p}<0,0001$ ) vezes maior de ter um adolescente reativo para IgG ou ambos, respectivamente, do que as outras unidades (Tabela 2).

Já entre os trabalhadores, 8 (7,9\%) voluntários apresentaram-se reagentes para $\operatorname{IgM,~} 6(5,9 \%)$ para IgG ou ambos, ao passo que $81(80,2 \%)$ participantes apresentaram-se não reagentes. Não foram observadas associações estatisticamente significativa entre a presença dos anticorpos e as unidades de trabalho dos participantes (Tabela 2).

Tabela 2: Associação entre localização da unidade e soroprevalência em adolescentes e trabalhadores.

\begin{tabular}{|c|c|c|c|c|}
\hline & $\begin{array}{c}\text { Capital } \\
(\text { Aracaju })\end{array}$ & $\begin{array}{c}\text { Outra cidade de } \\
\text { Sergipe (Nossa } \\
\text { Senhora do Socorro) }\end{array}$ & \multicolumn{2}{|c|}{ Teste exato de Fisher } \\
\hline $\begin{array}{l}\text { Adolescents } \\
\text { Units (n) }\end{array}$ & $\begin{array}{l}\text { Unidades 1-4 } \\
\quad(n=110)\end{array}$ & $\begin{array}{c}\text { Unidade } 5 \\
(n=43)\end{array}$ & $\begin{array}{c}\text { OR } \\
(\text { IC 95\%) }\end{array}$ & $\mathbf{p}$ \\
\hline IgM reagente & 8 & 2 & $\begin{array}{c}0,64 \\
(0,13-3,13)\end{array}$ & 0,73 \\
\hline IgG reagente & 26 & 22 & $\begin{array}{c}2,16 \\
(1,11-4,22)\end{array}$ & $0,03 *$ \\
\hline $\begin{array}{l}\operatorname{IgM} \text { e } \operatorname{IgG} \\
\text { reagente }\end{array}$ & 0 & 13 & $\begin{array}{c}68,59 \\
(3,99-118)\end{array}$ & $<0,0001 * * * *$ \\
\hline Workers (n) & $(n=60)$ & $(n=21)$ & $\begin{array}{c}\text { OR } \\
\text { IC 95\% }\end{array}$ & $\mathbf{p}$ \\
\hline IgM reagente & 5 & 3 & $\begin{array}{c}1,71 \\
(0,38-7,80)\end{array}$ & 0,68 \\
\hline IgG reagente & 3 & 3 & 2,86 & \\
\hline $\begin{array}{l}\operatorname{IgM} \text { e } \operatorname{IgG} \\
\text { reagente }\end{array}$ & 3 & 3 & $(0,53$ to 15,3$)$ & 0,34 \\
\hline
\end{tabular}

Fonte: Autores (2021).

A redução da propagação do vírus entre as pessoas infectadas é crucial para o combate à doença. Um estudo anterior do nosso grupo constatou que grande parte da população do Estado de Sergipe era assintomática para COVID-19, mas sorologicamente reativa para SARS-CoV-2 (Borges et al., 2020; Melo et al., 2020). Portanto, a identificação de anticorpos em amostras de sangue individuais, principalmente imunoglobulinas $\operatorname{IgM}$ e IgG, que são produzidas logo após a infecção, fornece um diagnóstico clínico da doença e permite seu monitoramento. Isso pode contribuir para uma redução na disseminação viral e mortalidade geral (Broadhurst et al., 2016; Jin et al., 2020; Kluge et al., 2018; Kraay et al., 2020). Dentre os testes sorológicos disponíveis, o método de imunofluorescência foi escolhido para os testes sorológicos realizados neste estudo (di Mauro et al., 2020; Lippi et al., 2020).

Deste modo, a investigação da soroprevalência de anticorpos IgM e IgG contra SARS-CoV-2 é um recurso complementar interessante (Borges et al., 2020; Zhang et al., 2020), a fim de verificar o grau de exposição e desenvolvimento de imunização na população e identificação de infecções assintomáticas (Long et al., 2020). No presente estudo, verificou-se a ocorrência de jovens e trabalhadores com anticorpos IgM e IgG, indicando a presença da doença e o desenvolvimento de imunidade contra ela, respectivamente.

De fato, foi documentado que IgM faz parte da linha inicial de defesa durante infecções virais e são mais úteis para determinar a infecção aguda, precedendo a formação de IgG que são importantes para a imunidade de longo prazo e a memória 
imune (Long et al., 2020; Racine \& Winslow, 2009). Além disso, com base no protocolo de diagnóstico e tratamento chinês para pneumonia por novo coronavírus (versão de teste 7), aproximadamente três a cinco dias após o início da infecção, um anticorpo IgM específico para SARS-CoV-2 torna-se detectável.

Já os níveis de anticorpos IgG são quatro vezes maiores durante a convalescença do que durante a fase aguda da doença. Em nosso estudo, anticorpos IgG foram detectados em $26(13,0 \%)$ adolescentes e $6(5,9 \%)$ trabalhadores. A presença de anticorpos IgG contra SARS-CoV-2 geralmente é indicativa de infecção atual ou anterior por SARS-CoV-2, e acredita-se que confira algum grau de imunidade (Azkur et al., 2020), porém a extensão e a duração do a imunidade não é clara. Em indivíduos que se recuperaram da doença, os anticorpos IgG persistem por pelo menos três meses (Guo et al., 2020; Long et al., 2020; Rodda et al., 2021). Mairesse et al. (2020) mostraram um aumento gradual nos anticorpos IgG anti-SARS-CoV-2 desde o início dos sintomas de COVID-19. Após 28 dias, foi observada uma fase de platô com ligeira diminuição dos níveis de IgG.

Além disso, observou-se a presença concomitante de $\operatorname{IgM}$ e $\operatorname{IgG}$ em 13 (6,5\%) adolescentes e 6 (5,9\%) trabalhadores, sendo possível sugerir a ocorrência de reinfecção (Bonifácio et al., 2020) ou sobreposição das curvas de produção das imunoglobulinas em questão (Long et al., 2020). De fato, os dados atuais sugerem que a soroconversão ocorre aproximadamente 7-14 dias após o início dos sintomas (Bohn et al., 2020), com aumento gradual no total de anticorpos desde o início dos sintomas de COVID-19 (Favresse et al., 2020).

Por fim, observou-se maior tendência à soropositividade entre os participantes da unidade 5 (Figura 1), sugerindo que a localização foi um importante determinante de saúde para a população deste estudo.

Figura 1: Tendência à soropositividade dos participantes da unidade 5 estudada.

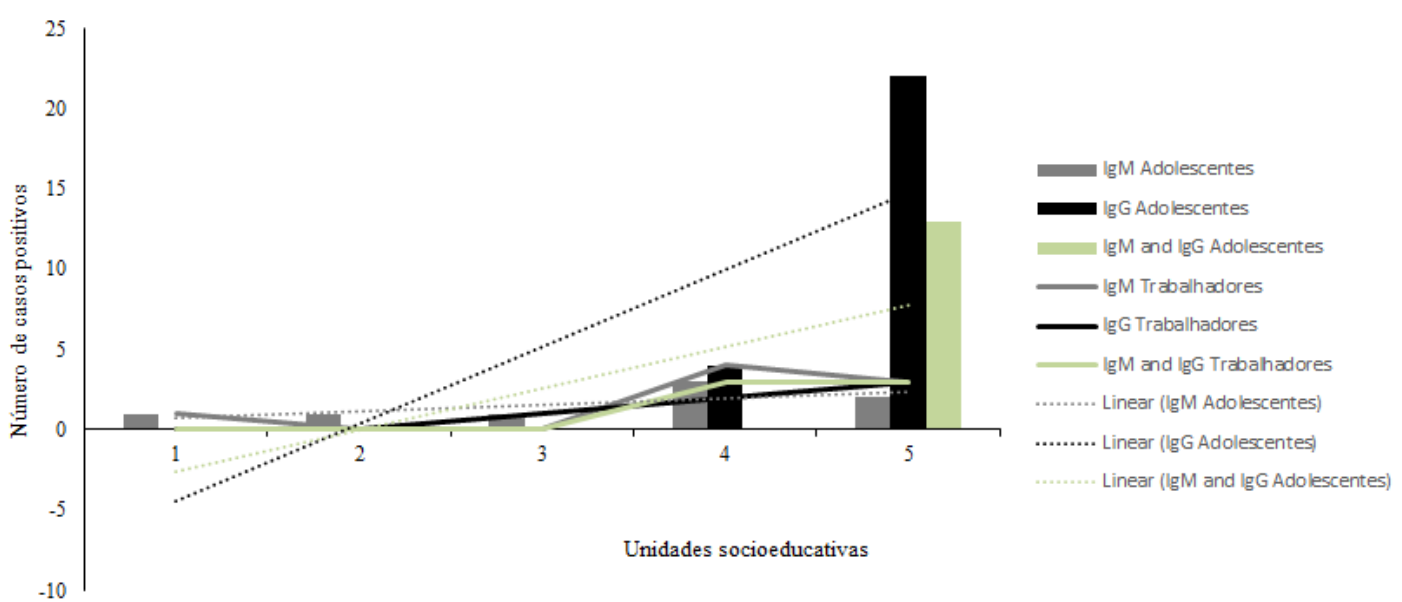

Fonte: Autores (2021).

De fato, entre as unidades participantes do estudo, percebeu-se que a de número 5 se destacou das demais, na qual os adolescentes apresentaram 2,16 e 68,59 vezes mais chances de ter IgG ou IgG e IgM, respectivamente, do que os adolescentes das unidades 1-4. A unidade denominada como 5 está localizada em outro município (Nossa Senhora do Socorro), no Estado de Sergipe, que fica a aproximadamente $17 \mathrm{Km}$ da capital Aracaju. $\mathrm{O}$ fato de adolescentes e trabalhadores dessa unidade apresentarem maior chance de ter anticorpos contra a SARS-CoV 2 pode estar relacionado às características estruturais das unidades. Ao contrário das unidades 1 a 4, onde o confinamento ocorre em quartos individuais, na unidade 5, cerca de oito adolescentes compartilham o mesmo dormitório (Figura 2), dificultando o isolamento social. Além disso, a adesão às medidas básicas recomendadas para prevenir a propagação do vírus, como uso de máscaras e higienização das mãos, é um desafio para 
as pessoas que vivem em confinamento. Sabe-se que as pessoas detidas apresentam maior risco de desenvolver doenças infecciosas contagiosas. Foi demonstrado no Brasil que viver em detenção aumenta o risco de tuberculose em 30 vezes (Mabud et al., 2019), e representa 17,5\% das mortes por doenças infecciosas em 2020 (Carvalho et al., 2020; Yang \& Thompson, 2020).

Figura 2: Ilustração das estruturas das unidades socioeducativas estudadas.

\section{Soroprevalencia contra SARS-CoV-2 em unidades socioeducativas de Sergipe, Brasil}

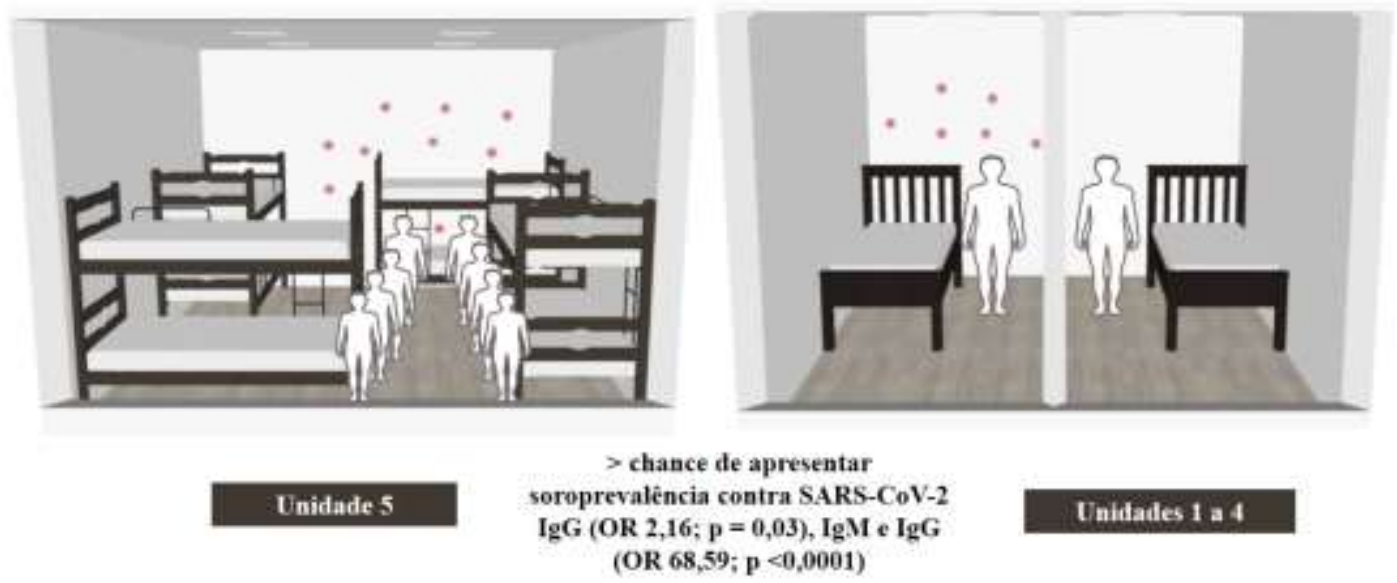

Fonte: Autores (2021).

Além disso, considerando a logística de funcionamento da Fundação Renascer do Estado de Sergipe, com regime de plantão e alta rotatividade de funcionários em cada unidade, e com alguns adolescentes podendo deixar as unidades nos finais de semana, é provável que a infecção tenha se disseminado na unidade por esses adolescentes e/ou por contato com funcionários assintomáticos infectados (os funcionários sintomáticos foram colocados em quarentena de acordo com as orientações da Organização Mundial de Saúde). Assim, é importante tomar medidas de combate à doença, como a testagem dos profissionais e dos adolescentes que têm permissão para sair da unidade; monitorar a adoção de medidas preventivas, como uso de máscaras, higienização das mãos, isolamento de pacientes sintomáticos ou positivos; bem como imunização.

\section{Conclusão}

Este estudo mostrou a prevalência de COVID-19 nas unidades de internação de adolescentes da Fundação Renascer no Estado de Sergipe, Brasil. Os testes realizados com o ensaio de imunofluorescência revelaram a taxa de anticorpos contra SARS-CoV-2 na população estudada e verificou-se que o tipo de confinamento parecia ser o determinante mais importante para a presença de $\mathrm{IgG}$ e coprevalência de $\operatorname{IgM}$ e IgG. Mesmo os adolescentes que não foram autorizados a sair das unidades apresentaram anticorpos anti-SARS-CoV-2. Estes achados demonstram a necessidade de ações efetivas para o controle da disseminação de doenças infectocontagiosas neste tipo de confinamento, bem como o desenvolvimento de Políticas Públicas eficazes para a assistência à saúde da população encarcerada no Brasil. Além disso, estes achados demonstram a necessidade da realização de mais estudos que avaliem as condições das unidades de encarceramento no Brasil, bem como apontem oportunidades de melhorias para possibilitar sistema de penalização mais humanizado e seguro. 
Research, Society and Development, v. 11, n. 1, e12611124680, 2022 (CC BY 4.0) | ISSN 2525-3409 | DOI: http://dx.doi.org/10.33448/rsd-v11i1.24680

\section{Agradecimentos}

Agradecemos à equipe da Fundação Renascer, ao Governo do Estado de Sergipe e à Universidade Federal de Sergipe pelo apoio concedido para a realização deste projeto.

\section{Referências}

Azkur, A. K., Akdis, M., Azkur, D., Sokolowska, M., van de Veen, W., Brüggen, M.-C., O’Mahony, L., Gao, Y., Nadeau, K., \& Akdis, C. A. (2020). Immune response to SARS-CoV-2 and mechanisms of immunopathological changes in COVID-19. Allergy, 75(7), 1564-1581. https://doi.org/10.1111/all.14364

Bohn, M. K., Lippi, G., Horvath, A., Sethi, S., Koch, D., Ferrari, M., Wang, C.-B., Mancini, N., Steele, S., \& Adeli, K. (2020). Molecular, serological, and biochemical diagnosis and monitoring of COVID-19: IFCC taskforce evaluation of the latest evidence. Clinical Chemistry and Laboratory Medicine, 58(7), 1037-1052. https://doi.org/10.1515/cclm-2020-0722

Bonifácio, L. P., Pereira, A. P. S., Araújo, D. C. de A. E., Balbão, V. da M. P., Fonseca, B. A. L. da, Passos, A. D. C., \& Bellissimo-Rodrigues, F. (2020). Are SARS-CoV-2 reinfection and Covid-19 recurrence possible? A case report from Brazil. Revista Da Sociedade Brasileira De Medicina Tropical, 53, e20200619. https://doi.org/10.1590/0037-8682-0619-2020

Borges, L. P., Martins, A. F., de Melo, M. S., de Oliveira, M. G. B., Neto, J. M. de R., Dósea, M. B., Cabral, B. C. M., Menezes, R. F., Santos, A. A., Matos, I. L. S., Borges, P. C., Dos Santos, K. A., Ribeiro, A. A., Menendez, A. I. M., Serafini, M. R., Walker, C. B., Quintans Junior, L. J., Araújo, A. A. de S., \& de Souza, D. R. V. (2020). Seroprevalence of SARS-CoV-2 IgM and IgG antibodies in an asymptomatic population in Sergipe, Brazil. Revista Panamericana De Salud Publica $=$ Pan American Journal of Public Health, 44, e108. https://doi.org/10.26633/RPSP.2020.108

Borges, L. P., Martins, A. F., de Souza, D. R. V., de Rezende Neto, J. M., Santos, A. A., Oliveira, B. M., Matos, I. L. S., da Invenção, G. B., dos Santos, K. A., Souza, N. A. A., de Jesus, P. C., dos Santos, C. A., de Oliveira Goes, M. A., de Souza, M. S. F., de Carvalho Barreto, I. D., Guimarães, A. G., \& QuintansJúnior, L. J. (2021). Does In-Person Visiting Affect the Number of COVID-19 Cases in Prisons? Life, 11(11), 1184. https://doi.org/10.3390/life11111184

Borges, L. P., Nascimento, L. C., Heimfarth, L., Souza, D. R. V., Martins, A. F., de Rezende Neto, J. M., Dos Santos, K. A., Matos, I. L. S., da Invenção, G. B., Oliveira, B. M., Santos, A. A., Souza, N. A. A., de Jesus, P. C., Dos Santos, C. A., Goes, M. A. O., de Souza, M. S. F., \& Guimarães, A. G. (2021). Estimated SARS-CoV-2 Infection and Seroprevalence in Firefighters from a Northeastern Brazilian State: A Cross-Sectional Study. International Journal of Environmental Research and Public Health, 18(15), 8148. https://doi.org/10.3390/ijerph18158148

Brasil. (2012). Conselho Nacional de Saúde. https://conselho.saude.gov.br/ultimas_noticias/2013/06_jun_14_publicada_resolucao.html

Brasil. (2021)._L12594.http://www.planalto.gov.br/ccivil_03/_ato2011-2014/2012/lei/112594.htm

Broadhurst, M. J., Brooks, T. J. G., \& Pollock, N. R. (2016). Diagnosis of Ebola Virus Disease: Past, Present, and Future. Clinical Microbiology Reviews, 29(4), 773-793. https://doi.org/10.1128/CMR.00003-16

Carvalho, S. G. de, Santos, A. B. S. dos, \& Santos, I. M. (2020). The pandemic in prison: Interventions and overisolation. Ciência \& Saúde Coletiva, 25, 3493-3502. https://doi.org/10.1590/1413-81232020259.15682020

CDC. (2020, fevereiro 11). Interim guidance on the management of coronavirus disease 2019 (COVID-19) in correctional and detention facilities. Centers for Disease Control and Prevention. https://www.cdc.gov/coronavirus/2019-ncov/community/correction-detention/guidance-correctional-detention.html

di Mauro, G., Scavone, C., Rafaniello, C., Rossi, F., \& Capuano, A. (2020). SARS-Cov-2 infection: Response of human immune system and possible implications for the rapid test and treatment. International Immunopharmacology, 84, 106519. https://doi.org/10.1016/j.intimp.2020.106519

Diamanti, K., \& Nikolaou, S. M. (2021). Researching the Social Impact of the COVID-19 Pandemic on Students in Greece. European Journal of Development Studies, 1(3), 26-32. https://doi.org/10.24018/ejdevelop.2021.1.3.35

Favresse, J., Eucher, C., Elsen, M., Laffineur, K., Dogné, J.-M., \& Douxfils, J. (2020). Response of anti-SARS-CoV-2 total antibodies to nucleocapsid antigen in COVID-19 patients: A longitudinal study. Clinical Chemistry and Laboratory Medicine, 58(10), e193-e196. https://doi.org/10.1515/cclm-2020-0962

Guo, L., Ren, L., Yang, S., Xiao, M., Chang, D., Yang, F., Dela Cruz, C. S., Wang, Y., Wu, C., Xiao, Y., Zhang, L., Han, L., Dang, S., Xu, Y., Yang, Q.-W., Xu, S.-Y., Zhu, H.-D., Xu, Y.-C., Jin, Q., ... Wang, J. (2020). Profiling Early Humoral Response to Diagnose Novel Coronavirus Disease (COVID-19). Clinical Infectious Diseases: An Official Publication of the Infectious Diseases Society of America, 71(15), 778-785. https://doi.org/10.1093/cid/ciaa310

Guo, W., Cronk, R., Scherer, E., Oommen, R., Brogan, J., Sarr, M., \& Bartram, J. (2019). A systematic scoping review of environmental health conditions in penal institutions. International Journal of Hygiene and Environmental Health, 222(5), 790-803. https://doi.org/10.1016/j.ijheh.2019.05.001

Huang, C., Wang, Y., Li, X., Ren, L., Zhao, J., Hu, Y., Zhang, L., Fan, G., Xu, J., Gu, X., Cheng, Z., Yu, T., Xia, J., Wei, Y., Wu, W., Xie, X., Yin, W., Li, H., Liu, M., ... Cao, B. (2020). Clinical features of patients infected with 2019 novel coronavirus in Wuhan, China. Lancet (London, England), 395(10223), 497506. https://doi.org/10.1016/S0140-6736(20)30183-5

Jin, Y., Wang, M., Zuo, Z., Fan, C., Ye, F., Cai, Z., Wang, Y., Cui, H., Pan, K., \& Xu, A. (2020). Diagnostic value and dynamic variance of serum antibody in coronavirus disease 2019. International Journal of Infectious Diseases: IJID: Official Publication of the International Society for Infectious Diseases, 94, 4952. https://doi.org/10.1016/j.ijid.2020.03.065

Kluge, H., Martín-Moreno, J. M., Emiroglu, N., Rodier, G., Kelley, E., Vujnovic, M., \& Permanand, G. (2018). Strengthening global health security by embedding the International Health Regulations requirements into national health systems. BMJ Global Health, 3(Suppl 1), e000656. https://doi.org/10.1136/bmjgh-2017-000656 
Kraay, A. N. M., Nelson, K. N., Zhao, C. Y., Demory, D., Weitz, J. S., \& Lopman, B. A. (2020). Modeling serological testing to inform relaxation of social distancing for COVID-19 control. medRxiv, 2020.04.24.20078576. https://doi.org/10.1101/2020.04.24.20078576

Lippi, G., Salvagno, G. L., Pegoraro, M., Militello, V., Caloi, C., Peretti, A., Gaino, S., Bassi, A., Bovo, C., \& Lo Cascio, G. (2020). Assessment of immune response to SARS-CoV-2 with fully automated MAGLUMI 2019-nCoV IgG and IgM chemiluminescence immunoassays. Clinical Chemistry and Laboratory Medicine, 58(7), 1156-1159. https://doi.org/10.1515/cclm-2020-0473

Long, Q.-X., Liu, B.-Z., Deng, H.-J., Wu, G.-C., Deng, K., Chen, Y.-K., Liao, P., Qiu, J.-F., Lin, Y., Cai, X.-F., Wang, D.-Q., Hu, Y., Ren, J.-H., Tang, N., Xu, Y.-Y., Yu, L.-H., Mo, Z., Gong, F., Zhang, X.-L., ... Huang, A.-L. (2020). Antibody responses to SARS-CoV-2 in patients with COVID-19. Nature Medicine, 26(6), 845-848. https://doi.org/10.1038/s41591-020-0897-1

Long, Q.-X., Tang, X.-J., Shi, Q.-L., Li, Q., Deng, H.-J., Yuan, J., Hu, J.-L., Xu, W., Zhang, Y., Lv, F.-J., Su, K., Zhang, F., Gong, J., Wu, B., Liu, X.-M., Li, J.-J., Qiu, J.-F., Chen, J., \& Huang, A.-L. (2020). Clinical and immunological assessment of asymptomatic SARS-CoV-2 infections. Nature Medicine, 26(8), 1200-1204. https://doi.org/10.1038/s41591-020-0965-6

Mabud, T. S., Alves, M. de L. D., Ko, A. I., Basu, S., Walter, K. S., Cohen, T., Mathema, B., Colijn, C., Lemos, E., Croda, J., \& Andrews, J. R. (2019). Evaluating strategies for control of tuberculosis in prisons and prevention of spillover into communities: An observational and modeling study from Brazil. PLOS Medicine, 16(1), e1002737. https://doi.org/10.1371/journal.pmed.1002737

Madahar, P., Wunsch, H., Jha, P., Slutsky, A. S., \& Brodie, D. (2021). Trends in COVID-19-related in-hospital mortality: Lessons learned from nationwide samples. The Lancet Respiratory Medicine, 9(4), 322-324. https://doi.org/10.1016/S2213-2600(21)00080-1

Mairesse, A., Favresse, J., Eucher, C., Elsen, M., Tré-Hardy, M., Haventith, C., Gruson, D., Dogné, J.-M., Douxfils, J., \& Göbbels, P. (2020). High clinical performance and quantitative assessment of antibody kinetics using a dual recognition assay for the detection of SARS-CoV-2 IgM and IgG antibodies. Clinical Biochemistry, 86, 23-27. https://doi.org/10.1016/j.clinbiochem.2020.08.009

Maruschak, L. M., Sabol, W. J., Potter, R. H., Reid, L. C., \& Cramer, E. W. (2009). Pandemic influenza and jail facilities and populations. American Journal of Public Health, 99 Suppl 2, S339-344. https://doi.org/10.2105/AJPH.2009.175174

Melo, M. S. de, Borges, L. P., Souza, D. R. V. de, Martins, A. F., Neto, J. M. de R., Ribeiro, A. A., Santos, A. A., Invenção, G. B. da, Matos, I. L. S., Santos, K. A. dos, Souza, N. A. A., Borges, P. C., \& Oliveira, M. G. B. de. (2020). Anti-SARS-CoV-2 IgM and IgG antibodies in health workers in Sergipe, Brazil (p. 2020.09.24.20200873). https://doi.org/10.1101/2020.09.24.20200873

Racine, R., \& Winslow, G. M. (2009). IgM in microbial infections: Taken for granted? Immunology Letters, 125(2), 79-85. https://doi.org/10.1016/j.imlet.2009.06.003

Rodda, L. B., Netland, J., Shehata, L., Pruner, K. B., Morawski, P. A., Thouvenel, C. D., Takehara, K. K., Eggenberger, J., Hemann, E. A., Waterman, H. R., Fahning, M. L., Chen, Y., Hale, M., Rathe, J., Stokes, C., Wrenn, S., Fiala, B., Carter, L., Hamerman, J. A., ... Pepper, M. (2021). Functional SARS-CoV-2Specific Immune Memory Persists after Mild COVID-19. Cell, 184(1), 169-183.e17. https://doi.org/10.1016/j.cell.2020.11.029

von Elm, E., Altman, D. G., Egger, M., Pocock, S. J., Gøtzsche, P. C., Vandenbroucke, J. P., \& STROBE Initiative. (2008). The Strengthening the Reporting of Observational Studies in Epidemiology (STROBE) statement: Guidelines for reporting observational studies. Journal of Clinical Epidemiology, 61(4), 344349. https://doi.org/10.1016/j.jclinepi.2007.11.008

WHO. (2020). Pneumonia of unknown cause - China. https://www.who.int/emergencies/disease-outbreak-news/item/2020-DON229

Yang, H., \& Thompson, J. R. (2020). Fighting covid-19 outbreaks in prisons. BMJ, 369, m1362. https://doi.org/10.1136/bmj.m1362

Zhang, J., Zhang, X., Liu, J., Ban, Y., Li, N., Wu, Y., Liu, Y., Ye, R., Liu, J., Li, X., Li, L., Qin, X., \& Zheng, R. (2020). Serological detection of 2019-nCoV respond to the epidemic: A useful complement to nucleic acid testing. International Immunopharmacology, 88, 106861. https://doi.org/10.1016/j.intimp.2020.106861

Zhu, H., Wang, L., Fang, C., Peng, S., Zhang, L., Chang, G., Xia, S., \& Zhou, W. (2020). Clinical analysis of 10 neonates born to mothers with 2019-nCoV pneumonia. Translational Pediatrics, 9(1), 51-60. https://doi.org/10.21037/tp.2020.02.06 